\title{
Origin of radially increasing stellar scaleheight in a galactic disk
}

\author{
C. A. Narayan and C. J. Jog
}

Department of Physics, Indian Institute of Science, Bangalore 560 012, India

e-mail: cjjog@physics.iisc.ernet.in

Received 29 April 2002 / Accepted 26 June 2002

\begin{abstract}
For the past twenty years, it has been accepted that the vertical scaleheight of the stellar disk in spiral galaxies is constant with radius. However, there is no clear physical explanation for this in the literature. Here we calculate the vertical stellar scaleheight for a self-gravitating stellar disk including the additional gravitational force of the $\mathrm{HI}$ and $\mathrm{H}_{2}$ gas and the dark matter halo. We apply our model to two edge-on galaxies, NGC 891 and NGC 4565, and find that the resulting scaleheight shows a linear increase of nearly a factor of two within the optical disk for both these galaxies. Interestingly, we show that the observed data when looked at closely, do not imply a constant scaleheight but actually support this moderate flaring in scaleheight.
\end{abstract}

Key words. galaxies: fundamental parameters - galaxies: individual: NGC 891, NGC 4565 - galaxies: kinematics and dynamics - galaxies: photometry - galaxies: spiral - galaxies: structure

\section{Introdution}

In the pioneering studies on vertical luminosity distribution of edge-on stellar disks, van der Kruit \& Searle (1981a,b) found that the vertical scaleheight of stars is independent of radius in NGC 4244, NGC 5907, NGC 4565 and NGC 891. This was later confirmed for NGC 4244 by Fry et al. (1999) and for NGC 891 by Kylafis \& Bahcall (1987) and also for other galaxies. In fact, the constant stellar scaleheight has become a wellestablished paradigm. Recent studies, however, have shown (Kent et al. 1991; de Grijs \& Peletier 1997) a moderate increase with radius in stellar scaleheight.

The constancy of scaleheight comes about when the scalelength with which the vertical velocity dispersion falls off exponentially along the radius, $h_{\mathrm{vel}}$, is twice the radial density scalelength, $h_{\mathrm{R}}$, in a galactic disk (van der Kruit \& Searle 1981a). However, this relation has no theoretical basis and is used only to obtain the flat scaleheight. We note that if the velocity falls off slower than the above rate $\left(h_{\mathrm{vel}}>2 h_{\mathrm{R}}\right)$, then it results in a flaring disk whereas the converse $\left(h_{\mathrm{vel}}<2 h_{\mathrm{R}}\right)$ gives rise to a disk which tapers down in thickness. These results are valid when the density distribution is defined only by the gravitational force of the stellar disk.

In reality, the molecular and atomic hydrogen gas and the dark matter halo exert a considerable gravitational force on the stellar disk and hence should be included in defining the stellar scaleheight. The effect of these components gains importance at large galactocentric radii, where the surface densities of all the components are comparable to each other, whereas

Send offprint requests to: C. A. Narayan, e-mail: chaitra@physics.iisc. ernet.in in the inner galactic disk the stellar component dominates over the others. We show that it is because of this additional gravitational force, that the stellar scaleheight is not exponentially flaring at large radii but instead it shows a controlled linear variation. Earlier we have proposed (Narayan \& Jog 2002) a gravitationally coupled, three-component disk plus halo model which predicts the scaleheight curves of $\mathrm{HI}, \mathrm{H}_{2}$ and the stellar disk of our Galaxy, which are shown to be in good agreement with observations.

In this Letter, we apply the same model for two external galaxies, NGC 891 and NGC 4565, and compare the stellar scaleheights obtained with observations available in the literature. These galaxies are chosen because these are two of the prototypical edge-on galaxies from the sample of van der Kruit \& Searle (1981a,b) which also have detailed $\mathrm{HI}$ and $\mathrm{H}_{2}$ surface density values from observations.

In Sect. 2, we discuss the details of the model used. The resulting scaleheights for NGC 891 and NGC 4565 are compared with observations in Sect. 3. Section 4 contains discussion and Sect. 5 gives a summary of the conclusions.

\section{Calculation for vertical stellar scaleheight}

The treatment for obtaining the vertical stellar scaleheight is based on a three-component disk plus halo model, as described by Narayan \& Jog (2002), which is briefly summarized below.

The force equation along the $z$-axis or the equation of hydrostatic equilibrium is given by (e.g., Rolhfs 1977):

$$
\frac{\left\langle\left(v_{z}\right)_{i}^{2}\right\rangle}{\rho_{i}} \frac{\mathrm{d} \rho_{i}}{\mathrm{~d} z}=\left(K_{z}\right)_{\mathrm{s}}+\left(K_{z}\right)_{\mathrm{HI}}+\left(K_{z}\right)_{\mathrm{H}_{2}}+\left(K_{z}\right)_{\mathrm{DM}}
$$


where $\rho$ is the mass density, $\left(K_{z}\right)=-\partial \psi / \partial z$ is the force per unit mass along the $z$-axis, $\psi$ is the corresponding potential, and the subscript $i=\mathrm{s}, \mathrm{HI}, \mathrm{H}_{2}$ and DM denotes these quantities for stars, $\mathrm{HI}, \mathrm{H}_{2}$ and the dark matter halo respectively. We take the root mean square of the vertical velocities of a component $\left\langle\left(v_{z}\right)_{i}^{2}\right\rangle^{\frac{1}{2}}$ or the random velocity dispersion at a radius $R$ and treat the component as being isothermal along $z$. The right hand side of Eq. (1) gives the total vertical force due to all the components.

For a thin disk, the joint Poisson equation reduces to :

$\frac{\mathrm{d}^{2} \psi_{\mathrm{s}}}{\mathrm{d} z^{2}}+\frac{\mathrm{d}^{2} \psi_{\mathrm{HI}}}{\mathrm{d} z^{2}}+\frac{\mathrm{d}^{2} \psi_{\mathrm{H}_{2}}}{\mathrm{~d} z^{2}}=4 \pi G\left(\rho_{\mathrm{s}}+\rho_{\mathrm{HI}}+\rho_{\mathrm{H}_{2}}\right)$

The coupled Eqs. (1) and (2) are solved for the density $\rho$ versus $z$, as a boundary value problem using standard numerical techniques (Press et al. 1986). To obtain the vertical density distribution of a component, we need to know the surface density of the component which is used as a boundary condition, and its vertical velocity dispersion. This results in a sech ${ }^{2}$-like density profile, and we use its $H W H M$ to define the scaleheight. We have used a single stellar component for simplicity, though recent observations show (de Grijs et al. 1997) a more sharply peaked luminosity profile which may be explained as a sum of several disk components.

\section{Results}

\subsection{NGC 891}

\subsubsection{Scaleheights from our model}

NGC 891 is an edge-on spiral $\left(i=89^{\circ}\right)$ of Hubble type Sb. It has a very large stellar disk with $h_{\mathrm{R}}=4.9 \mathrm{kpc}$ (van der Kruit $\&$ Searle 1981b). We use a central surface density of $700 M_{\odot} \mathrm{pc}^{-2}$ which is the value given for our Galaxy (Binney \& Tremaine 1987), for the sake of simplicity and also because the results from this section do not depend sensitively on the actual choice of the value used.

Bottema (1993) has measured the vertical velocity dispersions for a set of 12 spirals and finds that the central velocity dispersion is about $60 \mathrm{~km} \mathrm{~s}^{-1}$ for an Sb type galaxy, which is what we use for NGC 891 and NGC 4565. The dispersion falls off exponentially and is generally modeled (Lewis et al. 1989) with a scalelength, $h_{\mathrm{vel}}=2 h_{\mathrm{R}}$ in accordance with the van der Kruit \& Searle (1981a) model - see Sect. 1. A unique value of $h_{\mathrm{vel}}$ cannot be derived since there are only three data points for NGC 891 . Instead, in our work the ratio $h_{\mathrm{vel}} / h_{\mathrm{R}}$ is allowed to take a range of values between 1-4.

The observed surface densities of $\mathrm{HI}$ and $\mathrm{H}_{2}$ are taken from Rupen (1991) and Sofue \& Nakai (1993) respectively. The gas velocity dispersions in the Galaxy are $8 \mathrm{kms}^{-1}$ (Spitzer 1978) and $5 \mathrm{kms}^{-1}$ (Stark 1984; Clemens 1985), for $\mathrm{HI}$ and $\mathrm{H}_{2}$ respectively. These are taken to be the same for all the spiral galaxies, as observed for $\mathrm{HI}$ (Lewis 1984) and $\mathrm{H}_{2}$ (Wilson \& Scoville 1990) and as argued theoretically for $\mathrm{H}_{2}$ (Jog \& Ostriker 1988). Finally, we calculate the gravitational force of the dark matter halo from a standard model of Mera et al. (1998) with a total halo mass of $\sim 10^{12} M_{\odot}$. These input parameters are used to solve the coupled Eqs. (1) and (2).

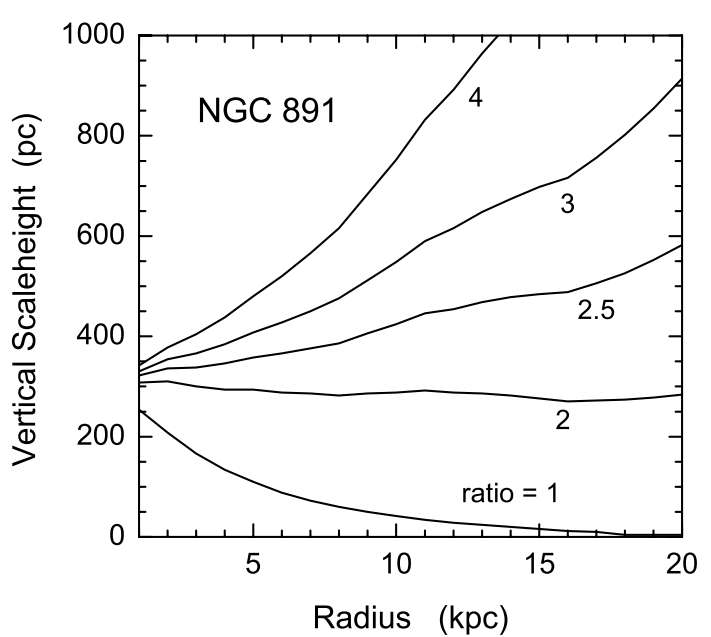

Fig. 1. Variation in resulting stellar vertical scaleheight with galactocentric radius for NGC 891 . The variation is found to crucially depend on the ratio $h_{\mathrm{vel}} / h_{\mathrm{R}}$. While the scaleheight is found to decrease by a factor of 50 and 1.1 for a ratio of 1 and 2 respectively, and increase by a factor of 1.8, 2.8 and 4.5 for the ratio of $2.5,3$ and 4 respectively, within the optical disk $(\leq 20 \mathrm{kpc})$. Thus the scaleheight need not be constant with radius for the galaxy.

Figure 1 shows the scaleheights obtained by the method discussed in Sect. 2 for the ratio $h_{\mathrm{vel}} / h_{\mathrm{R}}=1,2,2.5,3$ and 4 . This figure shows that in general, the scaleheight is not flat as deduced by van der Kruit \& Searle (1981b). The ratio of less than 2 can be ruled out since it gives unphysically tapering scaleheight.

\subsubsection{Scaleheights deduced from observed data}

Van der Kruit \& Searle (1981b) fit their data using an isothermal stellar disk model with the following space-luminosity density profile as applicable for an edge-on system:

$\mu(R, z)=\mu(0,0)\left(\frac{R}{h_{\mathrm{R}}}\right) K_{1}\left(\frac{R}{h_{\mathrm{R}}}\right) \operatorname{sech}^{2}\left(\frac{z}{z_{\mathrm{o}}}\right)$

where $\mu(0,0)$ is the space-luminosity density at the centre of the galaxy, $z_{\mathrm{o}}$ represents the scaleheight and $K_{1}$ is the modified Bessel function of the second kind. We note that, by definition, the scaleheight we obtain (see Sect. 2) is not the same as $z_{0}$ but the two are equivalent. This model gives a single and well-defined $\operatorname{sech}^{2}$ curve on a composite $z$-profile, when the $z_{\mathrm{o}}$ is kept constant. Figure 2 shows the composite $z$-profile for NGC 891. The solid line results when $z_{\mathrm{o}}=1 \mathrm{kpc}$ is kept constant with radius. However, we note that the observed data are spread over an interval about this single curve. We show that a similar spread in the calculated values of surface brightness is obtained when $z_{\mathrm{o}}$ is allowed to increase linearly from $0.75 \mathrm{kpc}$ to $1.25 \mathrm{kpc}$ over 4 disk scalelengths or the entire optical disk. This range of $z_{\mathrm{o}}$ is found by trial and error such that the model values match well with observations. This shows that the spread in the observed data allows for a linear increase of a factor of 1.7 in $z_{0}$. This increase allows us to constrain the value of $h_{\mathrm{vel}} / h_{\mathrm{R}}$ to be between 2-2.5 (see Fig. 1). 


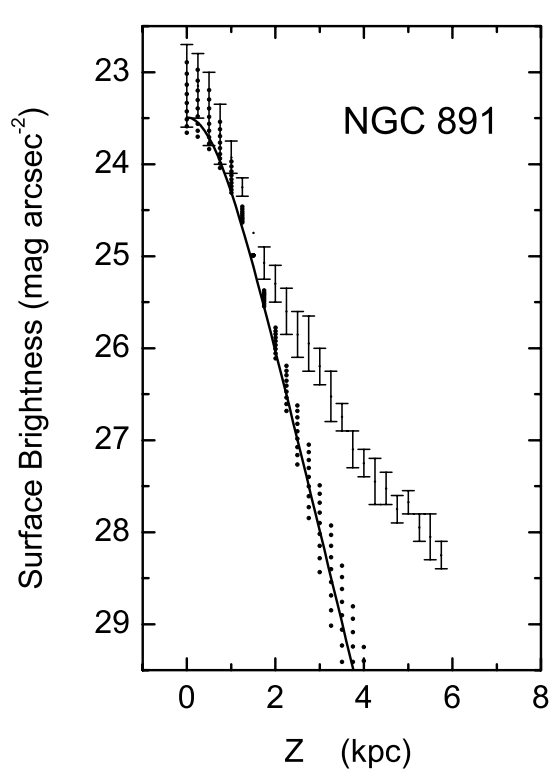

Fig. 2. The plot of surface brightness, $\mu$, versus the distance from the midplane, $z$, for NGC 891 . This composite $z$-profile is obtained by vertically shifting the individual $z$-profiles into coincidence at $z=1.5 \mathrm{kpc}$. The solid line results when $z_{0}=1 \mathrm{kpc}$ and is independent of radius. When $z_{\mathrm{o}}$ is linearly increased from $0.75-1.25 \mathrm{kpc}$ within $20 \mathrm{kpc}$, surface brightness values (points) disperse over an interval of $1 \mathrm{mag} / \mathrm{arcsec}^{2}$ at $z=0$, as is exactly seen in the original data (van der Kruit \& Searle 1981b). The vertical bars indicate the range of the data. This shows that the data can allow for as much as a factor of 1.7 increase in scaleheight. The deviation of the observed data at high $z$ is due to the thick disk which is not included in the model.

\subsection{NGC 4565}

NGC 4565 is an Sb type spiral galaxy with an inclination of $86^{\circ}$. Figure 3 shows the stellar scaleheight obtained for NGC 4565 using the method described in Sect. 2. The input parameters used here are as follows. The radial scalelength, $h_{\mathrm{R}}$ is $5.5 \mathrm{kpc}$ (van der Kruit \& Searle 1981a). The central surface density of stars is taken to be $700 M_{\odot} \mathrm{pc}^{-2}$ and the central vertical velocity dispersion $=60 \mathrm{kms}^{-1}$. The choice of above two numbers is justified in Sect. 3.1.1. Surface densities of HI and $\mathrm{H}_{2}$ are taken from Rupen (1991) and Sofue \& Nakai (1994) respectively. Since the observations for the stellar velocity dispersion are not available for NGC 4565 , we vary the ratio $h_{\text {vel }} / h_{\mathrm{R}}$ within the range of $1-4$.

Using the composite $z$-profiles (see Sect. 3.1.2), the stellar scaleheight for NGC 4565 was shown to be constant at $z_{\mathrm{o}}=0.79 \mathrm{kpc}$ (van der Kruit \& Searle 1981a). This was later confirmed by Naslund \& Jorsater (1997). As in the case of NGC 891, the observed surface brightness values do not give rise to a sharp and single sech ${ }^{2}$ curve. Figure 4 shows the numerically obtained composite $z$-profile, and also the observed data. The noted spread in observed data can be reproduced by increasing the $z_{\mathrm{o}}$ linearly from $0.4 \mathrm{kpc}$ to $1.0 \mathrm{kpc}$ within the optical disk $(\leq 22 \mathrm{kpc})$. This shows that the observed data of NGC 4565 can hide as much as a factor of 2.5 variation in scaleheight. This allows us to constrain $h_{\mathrm{vel}} / h_{\mathrm{R}}$ to be between 2.5-3 (see Fig. 3).

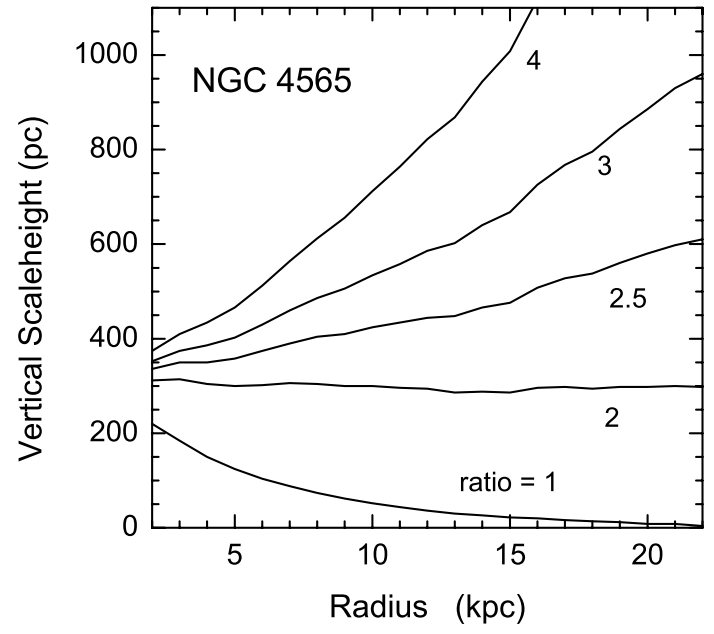

Fig. 3. Variation in resulting stellar vertical scaleheight with radius for NGC 4565. The scaleheight is found to decrease by a factor of 50 and 1.1 for a ratio of 1 and 2 respectively, and increase by a factor of 1.8 , 2.7 and 4.4 for the ratio of 2.5, 3 and 4 respectively, within the optical disk $(\leq 22 \mathrm{kpc})$. Thus the scaleheight need not be constant with radius for this galaxy too.

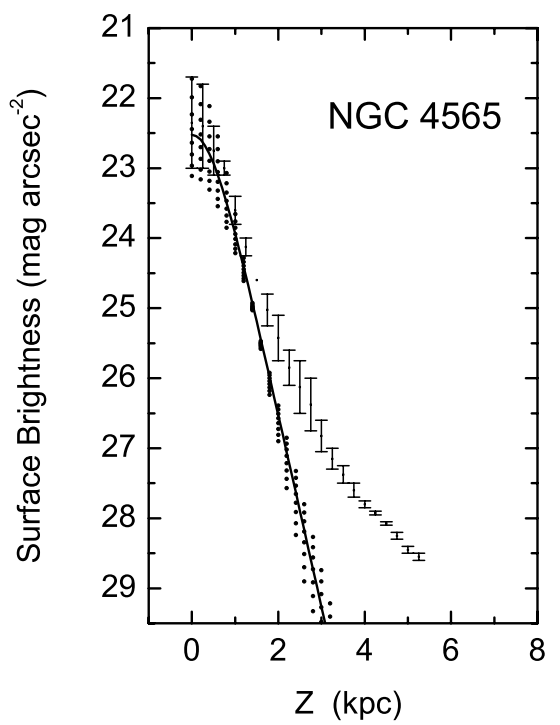

Fig. 4. The plot of surface brightness, $\mu$, versus the distance from the midplane, $z$, for NGC 4565, with details similar to Fig. 2 . The linear increase in $z_{\mathrm{o}}$ from $0.4-1.0 \mathrm{kpc}$ or an increase of a factor of 2.5 in the scaleheight can be easily hidden by the spread in the observed data.

\section{Discussion}

1. The results in Sect. 3 show that the observational data can accommodate a substantial radial variation in scaleheight. However, we would like to caution that the observed spread in data need not be entirely due to variation in scaleheight because, observational uncertainties such as the inclination, dust extinction and large measurement errors in photographic plates could also contribute to it. Morrison et al. (1997) conclude from a more complete analysis of the 2-D data that the scaleheight cannot be assigned a unique value, instead it lies within a range. This supports our model. 
2. From a recent study of 48 edge-on spirals, de Grijs \& Peletier (1997) find that the galaxies show a small radial increase in scaleheight in agreement with our model. They also find that the increase in scaleheight is smaller for later-type galaxies, and have explained this by using the presence of a thick disk. We note that, alternatively, this trend can be explained by our model. This is because there is an increase in the gas mass fraction (Binney \& Merrifield 1998) and the dark matter halo fraction (Broeils 1992) for later Hubble types. Therefore, their confining effect would be higher and hence the scaleheight increase would be smaller.

\section{Conclusions}

We examine the well-established constancy of vertical stellar scaleheight in spiral galaxies, and show that this result is not justified physically nor is it supported by observations. We show for NGC 891 and NGC 4565 that the observational uncertainties can hide as much as a factor of 2 increase in the scaleheight within the optical disk. We show that this variation can be explained theoretically by our model which takes account of the gravitational coupling between stars, gas, and the dark matter halo. Such moderate increase in scaleheight is likely to be common in other spiral galaxies as well.

Acknowledgements. Acknowledgements. We would like to thank the referee, R. de Grijs, for his detailed and constructive comments which have greatly improved the paper.

\section{References}

Binney, J., \& Tremaine, S. 1987, Galactic Dynamics (Princeton: Princeton Univ. Press)
Binney, J., \& Merrifield, M. 1998, Galactic Astronomy (Princeton: Princeton Univ. Press)

Bottema, R. 1993, A\&A, 275, 16

Broeils, A. H. 1992, Ph.D. Thesis, Groningen Univ.

Clemens, D. P. 1985, ApJ, 295, 422

de Grijs, R., \& Peletier, R. F. 1997, A\&A, 320, L21

de Grijs, R., Peletier, R. F., \& van der Kruit, P. C. 1997, A\&A, 327, 966

Fry, A. M., Morrison, H. L., Harding, P., \& Boroson, T. A. 1999, AJ, 118,1209

Jog, C. J., \& Ostriker, J. P. 1988, ApJ, 328, 404

Kent, S. M., Dame, T., \& Fazio, G. 1991, ApJ, 378, 131

Kylafis, N. D., \& Bahcall, J. N. 1987, ApJ, 317, 637

Lewis, B. M. 1984, ApJ, 285, L453

Lewis, J. R., \& Freeman, K. C. 1989, AJ, 97, 139

Mera, D., Chabrier, G., \& Schaeffer, R. 1998, A\&A, 330, 953

Morrison, H. L., Miller, E. D., Harding, P., Stinebring, D. R., \& Boroson, T. A. 1997, AJ, 113, 206

Narayan, C. A., \& Jog, C. J. 2002, A\&A, submitted

Naslund, M., \& Jorsater, S. 1997, A\&A, 325, 915

Press, W. H., Flannery, B. P., Teukolsky, S. A., \& Vetterling, W. T. 1986, Numerical Recipes (Cambridge: Cambridge Univ. Press), Chap. 6

Rohlfs, K. 1977, Lectures on Density Wave Theory (Berlin: SpringerVerlag)

Rupen, M. P. 1991, AJ, 103, 48

Sofue, Y., \& Nakai, N. 1993, PASJ, 45, 139

Sofue, Y., \& Nakai, N. 1994, PASJ, 46, 147

Spitzer, L. 1978, Physical Processes in the Interstellar Medium (New York: John Wiley)

Stark, A. A. 1984, ApJ, 281, 624

van der Kruit, P. C., \& Searle, L. 1981a, A\&A, 95, 105

van der Kruit, P. C., \& Searle, L. 1981b, A\&A, 95, 116

Wilson, C. D., \& Scoville, N. 1990, ApJ, 363, 435 